\title{
RADIO TELEMETRY DEVICES TO MONITOR BREATHING IN NON- SEDATED ANIMALS
}

\author{
Nathalie Samson, Sylvain Dumont, Marie-Laure Specq, Jean-Paul Praud \\ Neonatal Respiratory Research Unit, Departments of Pediatrics and Physiology, \\ Université de Sherbrooke, QC, Canada - J1H 5N4
}

Address for correspondence and proofs:

Jean-Paul Praud MD PhD

Departments of Pediatrics and Physiology

Université de Sherbrooke

J1H 5N4, QC Canada
Phone: 1 (819) 346-1110, ext 14851

Fax: $1(819)$ 564-5215

email: Jean-Paul.Praud@USherbrooke.ca 


\section{ABSTRACT}

Radio telemetry equipment has significantly improved over the last $10-15$ years and is increasingly being used in research for monitoring a variety of physiological parameters in non-sedated animals. The aim of this review is to provide an update on the current state of development of radio telemetry for recording respiration. Our literature review found only rare reports of respiratory studies via radio telemetry. Much of this article will hence report our experience with custom-built radio telemetry devices designed for recording respiratory signals, together with numerous other physiological signals in lambs. Our current radio telemetry system allows to record 24 simultaneous signals $24 \mathrm{~h} /$ day for several days. To our knowledge, this is the highest number of physiological signals, which can be recorded wirelessly. Our devices have been invaluable for studying respiration in our ovine models of preterm birth, reflux laryngitis, postnatal exposure to cigarette smoke, respiratory syncytial virus infection and nasal ventilation, all of which are relevant to neonatal respiratory problems.

Keywords: Wireless recording, polysomnography, lamb, apnea, sleep 


\section{INTRODUCTION}

Over the past few decades, scientists have adapted and developed, with the help of emerging technologies, various radio telemetry systems in their quest of studying and understanding biological functions. Radio telemetry is now considered state of the art in collecting numerous physiological signals in non-sedated, unrestrained animals, compared to conventional measurement techniques using hardwired equipment. It is generally acknowledged that the quality of physiological measurements collected from conscious, unstressed animals is superior, since they are collected under conditions that best represent the normal state of the animal and, when appropriate, are most predictive of the results that would be achieved in human beings (Kramer and Kinter, 2003).

Radio telemetry systems combine miniature sensors and transmitters to detect and transmit biopotentials in animals to remote receivers via an antenna. If necessary, the receiver converts the analog frequency signal into a digital signal to be computerized into a data acquisition system. There are several reliable, more or less invasive radio telemetry systems that are commercially available for simultaneous monitoring of up to 5 physiological signals in laboratory animals ${ }^{1}$. Radio telemetry systems for recording cardiovascular variables, including electrocardiogram (ECG), heart rate and blood pressure were initially developed for drug development companies (Brooks et al., 1996; Butz and Davisson, 2001; Kramer et al., 1993; Mills et al., 2000), in response to

${ }^{1}$ Data Science International, DSI (St-Paul, MN, USA); Emka Technologies (Paris, France); Konigsberg Instruments (Pasadena, CA, USA), Mini Mitter (Sunriver, OR, USA); Remo Technologies Ltd (Salisbury, UK); and Telemetronics Biomedical BV (Wageningen, The Netherlands) (list is not exhaustive). 
requirements by the FDA to submit continuous measurements of vital functions with each new drug application. Together with body temperature (Dilsaver et al., 1992) and activity indices (Mills et al., 2000), they have been the most frequently reported physiological signals measured via radio telemetry, especially in rodents. In addition, electroencephalogram (EEG) and electromyogram (EMG) have been recorded in a number of animal species, including mice, rats, rabbits, cats, dogs, sheep and monkeys (Antier et al., 1998; Champeroux et al., 1994; Herzog et al., 1993; Kant et al., 1995; Kawai et al., 2008; Letourneau et al., 1999; Letourneau and Praud, 2003; Livezey and Sparber, 1990; Meile and Zittel, 2002; Mumford and Wetherell, 2001; Nakajima et al., 1996; Sei and Morita, 1996; Tang et al., 2005). However, until very recently, the use of telemetry for studying respiration was restricted to a handful of reports on respiratory rate derived from pleural pressure (Murphy et al., 1998) or arterial pressure waveforms (reviewed in Kramer and Kinter, 2003).

A number of respiratory signals can theoretically be considered for inclusion in a radio telemetry system designed for recording breathing in animals (Baekey et al., 2009; AlKhalidi et al., 2011). Airflow limitations and/or apneas can be identified using nasal thermistor/thermocouple, nasal pressure cannula, nasal capnography, nasal or facial mask pneumotachography or tracheal sounds via a microphone or a piezoelectric sensor attached on the upper thorax or neck. Recording of rib cage and abdominal movements (strain gauge or piezoelectric belts, impedancemetry, inductance plethysmography), as well as respiratory efforts (diaphragm electromyography, pleural or esophageal pressure) allows identification of central apneas. In addition, when used with a sensor aimed at recognizing airflow limitation/apnea recording, such respiratory 
signals allow apnea characterization as central, obstructive or mixed. Pulse oximetry and $\mathrm{PCO}_{2}$ monitoring, either via end tidal capnography or transcutaneously, are unique indices of efficacious breathing. Overall, while respiratory frequency can be derived from every respiratory signal collected, tidal volume can only be measured by calibrated impedance plethysmography, pneumotachograph and by respiratory metabolic plethysmography chamber in small rodents. Each technique has its own distinct advantages and limitations and should be chosen according to the research question, the animal species and the experimental conditions. Also, in selected cases, it is relevant to consider recording of respiratory signals such as electrical activity of thoracic, abdominal or upper airway muscles.

To our knowledge, it is only very recently that wireless recording of some respiratory signals has been made commercially available (see table 1). Our group has been actively studying the perinatal control of breathing in lambs since the late 80's, in an attempt to better understand the pathophysiology of apneas of prematurity, apparent-life threatening events and sudden infant death syndrome, with a special focus on laryngeal physiology (Praud et al., 2006; Samson et al., 2007, 2008; St-Hilaire et al., 2007). Although rapidly developing, the sophisticated telemetry systems at the time did not allow respiration studies. For the purpose of our research needs, it became necessary to design and build our own radio telemetry system to allow for simultaneous recording of numerous biopotentials linked to respiration during prolonged polysomnographic recordings in non-sedated, freely moving lambs. The present article provides an overview of our telemetered recording systems and reports a few examples of the data 
obtained in our various lamb models, while underscoring some limitations and our attempts at improvement. 


\section{DESCRIPTION OF OUR TELEMETERED RECORDING SYSTEM}

\subsection{SURGICAL INSTRUMENTATION AND RECORDING ELECTRODES / PROBES}

Chronic surgical instrumentation under general anesthesia ( $2 \%$ isoflurane, $30 \% \mathrm{~N}_{2} \mathrm{O}$, $68 \% \mathrm{O}_{2}$ ) is performed in the first few days of life in all lambs, in order to implant indwelling catheters (for monitoring fluid or air pressures) and electrodes (for monitoring biopotentials). Over the years, we have developed our own recording electrodes and indwelling catheters. As previously described, two needle-electrodes are inserted into the parietal cortex directly through the skull for electrocorticogram (ECoG) recording, while a third needle-electrode is inserted under the scalp as a ground (Carreau et al., 2011). Eye movements (EOG) are recorded with two custom-built silver electrodes inserted subcutaneously close to the right eye socket. The electrocardiogram is recorded with 2 needle-electrodes inserted under the periosteum of the $5^{\text {th }}$ rib, on both sides of the thorax, and directly glued on the rib (Carreau et al., 2011). Respiratory muscle EMG activities (including diaphragm, glottal and/or pharyngeal muscles) are recorded with custom-built bipolar electrodes, made from either right or $180^{\circ}$ angled gold connectors (Sullins Connector Solutions, Digi-Key Corporation, Thief River Falls, MN, USA). These EMG electrodes are inserted perpendicularly into the muscular fibers and then secured with a stitch. All indwelling catheters are made from silastic tubing (0.03 in. ID, Dow Corning, Midland, MI) and inserted directly into either the carotid artery (arterial pressure measurements) or between two tracheal rings (respiratory pressure measurements). Leads from all electrodes are subcutaneously tunneled to a common exit on the lamb's back. All recording electrodes and catheters are protected by 
bandages and by a jacket worn by the lamb. Immediately prior to the recordings, further external instrumentation is added, including a J-type thermocouple for nasal flow recording, thoracic and abdominal elastic bands for respiratory inductance plethysmography and a Masimo pulse oximeter probe on the tail root. During polysomnographic recordings, all catheters, electrodes and probes are connected to our radio telemetry system housed in the lamb's jacket. All procedures described, including surgical instrumentation and subsequent polysomnographic recordings in lambs, have been approved by the Ethics Committee on Animal Care and Experimentation of our institution.

\subsection{RADIO TELEMETRY SYSTEM}

Our radio telemetry system can simultaneously transmit and record up to 24 physiological signals of interest for the study of sleep apneas in freely moving lambs. These parameters include ECoG and EOG for scoring states of alertness, upper airway and respiratory muscle EMGs, respiratory pressures, nasal flow and thoraco-abdominal movements for detection and characterization of apneas, as well as cardio-vascular data, such as ECG and blood pressure. Finally, we can also record arterial hemoglobin saturation in $\mathrm{O}_{2}$ using pulse oximetry $\left(\mathrm{SpO}_{2}\right)$.

\subsubsection{Radio telemetry transmitters}

Our current radio telemetry system is comprised of 3 different transmitters: 1) one 12channel analog transmitter for EMG (x 8), ECG, ECoG (x 2), EOG and ground; 2) one 8-channel digital transmitter for abdominal and thoracic movements, nasal flow (x 2) and respiratory or arterial pressures $(x 4)$; 3) one digital transmitter for $\mathrm{SpO}_{2}$, pulse rate, 
signal strength and plethysmographic signal. The transmitters are housed in a jacket with several pockets; each wire stemming from an implanted electrode or an external probe, as well as each catheter, is connected to its specific transmitter, while protected by the jacket in its external course.

Twelve-channel analog transmitter. In the past year, we have extended our initial 8channel analog radio telemetry system (Letourneau and Praud, 2003). Indeed, 4 additional biopotentials can now be collected with the latest version. The 12-channel analog transmitter (dimensions $63 \times 52 \times 4 \mathrm{~mm}$ ) is built using surface-mounted technology and a four-layer printed circuit board (PCB). Each channel has its own differential preamplifier, high-pass filter and low-pass filter, allowing gain and cutoff frequency of each channel to be specifically adjusted to the biopotential to be transmitted. The twelve channels include eight channels for EMG acquisition (frequency range: $30-500 \mathrm{~Hz}$, gain: 390$)$, two channels for ECoG acquisition $(0.16-500 \mathrm{~Hz}$, gain: 10) and two other channels (frequency range 1.6-500 Hz) for EOG (gain: 107) and ECG (gain: 10) acquisition (see block diagram in Figure1-A). The 12-channel radio telemetry system uses time division multiplexing to transmit all channels on the same carrier. The time division multiplexing assigns a different time interval of $70 \mu$ s to each channel. At the end of the $12^{\text {th }}$ channel, a synchronization interval of $140 \mu \mathrm{s}$, during which transmission is interrupted, is added to ensure synchronization between receiver and transmitter. All biopotentials are thus sampled at a rate of $1 \mathrm{KHz}$. Finally, the radiofrequency (RF) carrier is frequency-modulated by this resulting signal, which is called pulse amplitude modulation/time division multiplexing (PAM/TDM). The transmission frequency can be adjusted within the biomedical band range for Canada (176-216 
$\mathrm{MHz}$ ); the frequency used herein is $183 \mathrm{MHz}$. The transmitter antenna is $33.5 \mathrm{~cm}$ long and constructed with flexible wire (whip-type antenna). The system is designed to transmit biopotentials up to a maximal distance of 2 meters.

Eight-channel digital transmitter. The digital transmitter (dimensions $63 \times 51 \times 14$ $\mathrm{mm}$ ) is built using surface-mounted technology PCB (as described above). Each channel has its own differential preamplifier, high-pass and low-pass filter, allowing gain and cutoff frequency of each channel to be specifically adjusted to the biopotential to be transmitted. It comprises eight channels, including two channels for abdominal and thoracic movements (frequency range: $\mathrm{DC}-150 \mathrm{~Hz}$, gain: 300 ), two channels for nasal flow (frequency range: DC-150 Hz, gain: 1750) and four channels for respiratory or arterial pressure (frequency range: DC-150 Hz, gain: 500 and 1000) acquisition (see block diagram in Figure 1-B). The 8 channels are multiplexed and sequentially sampled by a 16-bit successive-approximation register (SAR) analog-to-digital converter (ADC) at a rate of 300 samples per second per channel. ADC results are then sent to the microcontroller which generates a frame comprising a framing byte (0xAA) followed by the 16-bit ADC result of each of the eight channels. The frame is then transmitted at a rate of $56 \mathrm{Kbps}$ using a frequency shift keying (FSK) transmitter operating at $916 \mathrm{MHz}$, which falls into the ISM (Industrial, Scientific and Medical) band for North America (902$928 \mathrm{MHz})$. The transmitter antenna used is an ultra compact omni directional antenna (ANT-916-JJB-RA, Antenna Factor, Merlin, OR). The system is designed to transmit biopotentials at a maximal distance of 10 meters.

Masimo pulse oximetry transmitter. The Masimo pulse oximetry transmitter (dimensions $63 \times 54 \times 11 \mathrm{~mm}$ ) is built using surface mounted technology and includes 
the MS-13 OEM Board from Masimo Corporation and the RF transmitter (see block diagram in Figure 1-C). It also includes a $5 \mathrm{~V}$ step-up DC/DC converter to power the $5 \mathrm{~V}$ based MS-13 OEM board from the 3.7 V Li-lon battery. A microcontroller is used to configure the MS-13 OEM board at power-up. The MS-13 OEM board then outputs a proprietary message at a rate of 9600 bps that is wirelessly transmitted using a FSK transmitter operating at $903 \mathrm{MHz}$, which falls into the ISM band for North America (902$928 \mathrm{MHz}$ ), via an ultra compact omni directional antenna (ANT-916-JJB-RA, Antenna Factor, Merlin, OR). The four signals transmitted are $\mathrm{SpO}_{2}$, heart rate, pulse wave and signal strength. The system is designed to transmit the MS-13 information at a maximal distance of 10 meters.

All transmitters are powered with an Ultralife Lithium-lon rechargeable battery offering a capacity of 900 mAh (UBP002, Ultralife Corporation, Newark, NY), which provides up to $16 \mathrm{~h}$ for both the 12-channel analog and the 8-channel digital transmitters and up to $8 \mathrm{~h}$ for the Masimo pulse oximetry transmitter. All transmitters are inserted into a custombuilt photopolymer case designed by stereolithography.

\subsubsection{Radio telemetry receivers}

Twelve-channel analog receiver. As previously described (Letourneau and Praud, 2003), the receiving antenna (RG-59, GE Marquette Canada, Mississauga, ON, Canada) is placed at about $2 \mathrm{~m}$ from the recorded lamb. The receiver demodulates, synchronizes and demultiplexes the frequency-modulated PAM/TDM signal in order to rebuild the original signals (Figure 2-A). The superheterodyne receiver provides the demodulated signal and a receiver signal strength indicator (RSSI). A sudden change in RSSI signal indicates the synchronization interval sent by the transmitter. This allows 
synchronization of the demultiplexer clock with that of the multiplexer (transmitter). The PAM/TDM signal can thus be demultiplexed by selecting the specific time interval associated with each individual channel and low-pass filtered to recover the original signal from each channel. For more flexibility, each channel has its own adjustable gain amplifier. Finally, a low output impedance buffer allows connection of the receiver outputs to a computer acquisition system (Biopac MP $150+$ AcqKnowledge software version, Biopac Systems, Goleta, CA, USA).

Eight-channel digital receiver. The demodulator unit of the $916 \mathrm{MHz}$ FSK receiver is combined with the antenna in the lamb's recording room. The demodulated RF signal is sent through an RS485 link to the reconstruction unit of the receiver located in an adjacent room. A microcontroller analyzes the received data to detect the presence of the framing byte (OXAA), identifying the beginning of a new frame (Figure 2-B). This allows the synchronization and demultiplexing of the data to the appropriate channel. The microcontroller then sequentially updates the 16-bit digital-to-analog converter (DAC) of each channel at a rate of 300 samples per second per channel. Each channel has its own adjustable gain amplifier and a $4^{\text {th }}$ order, $150 \mathrm{~Hz}$ low-pass filter to recover the original signal. Finally, a low output impedance buffer allows connection of the receiver outputs to the Biopac computer acquisition system.

Masimo pulse oximetry receiver. The demodulator unit of the $903 \mathrm{MHz}$ FSK receiver is combined with the antenna in the lamb's recording room. The demodulated RF signal is sent through an RS485 link to the reconstruction unit of the receiver located in an adjacent room. A microcontroller analyzes the received data to extract the desired information from the Masimo proprietary message (Figure 2-C). This allows extraction of 
the 8-bit autoscaled IR plethysmographic signal at a rate of $62.5 \mathrm{~Hz}$ and the $\mathrm{SpO}_{2}$, pulse rate and signal strength values at a rate of $1 \mathrm{~Hz}$. The microcontroller then sequentially updates the 16-bit digital-to-analog converter (DAC) of the corresponding channel at the appropriate rate. Each channel has its own adjustable gain amplifier. Additionally, the plethysmographic signal channel has a $4^{\text {th }}$ order, $31 \mathrm{~Hz}$ low-pass reconstruction filter. Finally, a low output impedance buffer allows connection of the receiver outputs to the Biopac computer acquisition system.

\subsection{VIDEO RECORDING}

Simultaneous video recordings are systematically performed to monitor the lamb's behavior in real time during polysomnographic recordings, using a webcam modified for infrared vision (Microsoft, Lifecam, VX-3000). Adequate infrared lighting is ensured by an infrared LED dome illuminator (CV-100 DE/DR, DLi Systems, Dubai, United Arab Emirates). The video files, which are synchronized with the other physiological data by the AcqKnowledge software, provide essential information, especially for recognizing states of alertness and characterizing position and activity.

\section{IN VIVO VALIDATION AND USE OF OUR RADIO TELEMETRY}

\section{SYSTEM}

In vivo validation of the accuracy of each data channel has been performed for the three radio telemetry transmitters. Indeed, data obtained with our 8/12-channel analog system $(E M G \times 4-8, E C o G \times 2$, EOG $\times 1, E C G \times 1)$ have been validated by comparing them with those simultaneously recorded using conventional, hardwired equipment (Letourneau and Praud, 2003). Results obtained with both systems were virtually 
identical, especially including sleep stage scoring, bradycardia detection and laryngeal/respiratory muscle EMG activity. In addition, data obtained with our 8-channel digital transmitter (thoracic and abdominal movements $\times 2$, nasal flow $\times 2$ and pressure x 4) were compared with results obtained with conventional, hardwired equipment. Accordingly, thoracic and abdominal movements were simultaneously recorded with the original, hardwired respiratory inductance plethysmography equipment (Respitrace, Monitoring systems, Miami Beach, FL, USA) and with our wireless system in 5 nonsedated newborn lambs. Results showed excellent and consistent concordance between both systems for apnea detection and characterization, together with relative variations in tidal volume amplitude. Nasal flow as well as respiratory or arterial pressures (RX104A pressure transducer, Biopac Systems, Goleta, CA, USA) were also compared and validated with standard, hardwired equipment. Indeed, nasal flow signal was compared with airflow collected using a pneumotachograph $(21070 \mathrm{~B}+47304 \mathrm{~A}$ flow transducer, Hewlett Packard, Hewlett-Packard Company, Palo Alto, CA, USA), whereas respiratory and arterial pressures were compared with calibrated pressure transducers (respectively Validyne, Northridge, CA and Trantec model 60-800, American Edwards Laboratories, Santa Anna, CA). Results obtained for each parameter were virtually identical to conventional measurements (unpublished data). Finally, validation of our wireless Masimo pulse oximeter was performed similarly to our previous wireless Nonin pulse oximeter system (Reix et al., 2005). Briefly, the sensors (LNOP YI reflectance sensor from Masimo, Irvine, CA) of both our wireless Masimo pulse oximeter and the standard hardwired Masimo Radical pulse oximeter were attached externally at the root of the lamb's tail for continuous monitoring of arterial 
$\mathrm{SpO}_{2}$, heart rate and pulse wave. Under various $\mathrm{O}_{2}$ conditions, our wireless pulse oximeter was as reliable as the standard Masimo pulse oximeter currently used in our laboratory, for monitoring and detecting variations in $\mathrm{SpO}_{2}$ variations (unpublished results).

Furthermore, after validation of the accuracy of each data channel, prolonged and repeated polysomnographic recordings were conducted in 17 lambs (including 7 preterm lambs), to test whether our telemetry system was well adapted to our needs for prolonged recordings. Continuous polysomnographic recordings (with battery changes every 8 to $12 \mathrm{~h}$ ) were performed over a period of up to 7 days, totaling more than $1200 \mathrm{~h}$ of recording. Overall, our radio telemetry system was shown to be particularly well suited for studying cardio-respiratory function during the various sleep states in our nonsedated, freely-moving preclinical ovine models, and over a period of at least 7 days. Examples of polysomnographic recordings obtained in newborn lambs using our radio telemetry system are illustrated in Figures 3, 4 and 5.

\section{DISCUSSION}

The present article reports the development and performance of a wireless radio telemetry system for prolonged polysomnographic recordings of respiration and related physiological signals in non-sedated, freely moving lambs. This comprehensive set of devices allows to perform a complete and extensive assessment of breathing during all states of alertness, including cardio-vascular consequences of apneas in large animals, in conditions as close to normal as possible. To our knowledge, it offers the highest number of physiological signals, which can be recorded simultaneously and wirelessly 
throughout the various states of alertness. Given that restraints prevent the normal cycling of states of alertness (Hegde et al., 2008; McLeod et al., 1979; Tang et al., 2007), and can disturb normal breathing (Al-Qarawi, 2005; DeLorme and Moss, 2002), such a system is an invaluable asset for a research program such as ours.

\subsection{IMPORTANCE OF TELEMETRY IN BIOMEDICAL RESEARCH}

Telemetry is now considered state of the art in collecting physiological signals in the absence of sedation, compared to conventional systems in which animals are hardwired to the recording devices, and consequently more stressed. In addition, telemetry allows us to reduce animal use by increasing the number of physiological signals collected, as well as the number of recording sessions in each animal. Hence, in addition to increasing the odds of recording normal physiology (Kramer et al., 2001), the use of telemetry in biomedical animal research also constitutes a major asset from an ethical standpoint. Indeed, the resulting decreased invasiveness, together with the need for fewer animals per experiment, clearly contributes to the refinement and reduction components of the now inevitable "3 Rs concept" (refinement, reduction, replacement) proposed 40 years ago by Russell and Burch (1959). Finally, the use of wireless telemetry substantially avoids wire setup breakage, which can be frequent with hardwired recording equipment. The success of radio telemetry, especially with drug development companies, has led several equipment manufacturers to enter and develop this specific market, mentioned in the introduction section.

\subsection{TELEMETERED RECORDING OF BREATHING}


As reviewed in the introduction section, an increasing number of physiological signals can now be recorded via telemetry. Interestingly, while the use of radio telemetry has initially been largely restricted to cardiovascular function in rodents, a clear trend in recording additional physiological functions, including respiration, and in applying telemetry to large animals, has appeared in the last decade.

Review of the literature reveals that only a limited number of research teams have recorded respiration, using either custom-built or commercially available wireless equipment. Microwave radiation recording of respiratory rate was first proposed in rodents (Gordon and Ali, 1984) and was used more recently in hibernating black bears (Suzuki et al., 2009). Impedance monitoring has recently been used in monkeys (Authier et al., 2010) and dogs (Kearney et al., 2010). Respiratory rate has also been derived from arterial pressure waveform in rodents (reviewed in Kramer and Kinter, 2003), and from pleural pressure in rats (Murphy et al., 1998), dogs (Ednick et al., 2007) and monkeys (Carrier et al., 2010; Layton et al., 2011; Warren et al., 2011). Respiratory inductive plethysmography has been used in dogs (Murphy et al., 2010). Finally, one group has reported respiratory rate recording using custom-built equipment, namely an acoustic sensor in swine and a thoracic or abdominal circumference in cattle (Eigenberg et al., 2008).

To our knowledge, wireless recording equipment of respiration integrated with other physiological signals can now be purchased from two manufacturers. The main differences with our system are presented in table 1. The most significant difference relates to the greater number of physiological signals, which can be recorded with our custom-built system (EEG, EOG, EMGs, nasal flow, $\mathrm{SpO}_{2}$, respiratory or arterial 
pressures), making it uniquely suited to polysomnographic recordings. In addition, our telemetry system can be used with any common data acquisition system, such as from Biopac Systems, AD Instruments or National Instruments. This flexibility is a unique advantage compared to commercially available telemetry systems, which work with their proprietary software and are often very difficult to interface with common data acquisition systems.

Throughout the years, our radio telemetry system has allowed us to study numerous physiological signals related to neonatal breathing. Beyond nasal flow (thermocouples) and thoraco-abdominal movements (respiratory inductance plethysmograph), we have been particularly interested in EMG activities of respiratory muscles and upper airway muscles. Thus, EMG of the diaphragm, laryngeal intrinsic muscles including the thyroarytenoid muscle (glottal constrictor) and the cricothyroid muscle (glottal dilator), as well as pharyngeal muscles such as the genioglossus and the levator palatini (figure 4) have been recorded under various conditions.

The contribution of our telemetry system has proven to be an invaluable tool for studying breathing anomalies in our various preclinical neonatal ovine models. These unique models include apneas in premature lambs (Renolleau et al., 1999; St-Hilaire et al., 2007), application of nasal CPAP (Samson et al., 2005, 2008) or intermittent positive pressure ventilation (Roy et al., 2008), postnatal exposure to cigarette smoke (St-Hilaire et al., 2010), reflux laryngitis (Carreau et al., 2011), respiratory syncytial virus infection (unpublished results), all of which are relevant to neonatal respiratory problems. Overall, review of the scientific literature suggests that our team has the most extensive and consistent publication track record on respiration recording via radio telemetry. 


\subsection{LIMITATIONS OF OUR TELEMETRY SYSTEM - THE NEED FOR CONTINUOUS IMPROVEMENT}

\subsubsection{Surgical chronic instrumentation: a special concern}

From our standpoint, while the jacket-worn transmitters fulfill the needs for minimal invasiveness (and increased possibility of reuse in a number of animals over several years), our current surgical, chronic instrumentation remains nevertheless too invasive. This is of special concern within the context of our research program focused on the early postnatal period. Indeed, in order to study this critical period, we are often obliged to start recordings as early as 2 days after the 2-3 hour-long surgery necessary to implant our indwelling catheters and electrodes, at a time where some post-surgery stress is still present and could interfere with the results. We believe that a significant improvement in our research design should come from the ability to decrease and even avoid surgical stress whenever possible. Some progress has been made in the last years to decrease this stress, e.g. by using thoracic videosurgery instead of conventional thoracotomy (Roy et al., 2008; Samson et al., 2008). However, we are continually committed to finding new ways to replace surgery by non-invasive instrumentation as often as possible, without decreasing the quality of recorded physiological signals. This is already virtually up and running for the respiratory component of our recordings, by using thermocouples glued on the skin for nasal flow, bands of respiratory inductance plethysmography stretched around the rib cage and abdomen for respiratory movements and an oximeter probe attached to the lamb's tail root for blood oxygenation. However, as already pointed out, an important aspect of our recordings is the ability to study respiration within a polysomnography setting. We are 
thus currently assessing the best means to continuously record and recognize the states of consciousness using electrophysiological signals (EEG, EOG) via subcutaneous, needle-electrodes or external cups glued on the skin vs. infrared video recordings in combination with EOG and regularity of respiration. Furthermore, we are currently testing the possibility to chronically instrument laryngeal muscles, as well as the crural diaphragm, using modified transcutaneous techniques (Insalaco et al., 1991; Trelease et al., 1982).

\subsubsection{Lingering technical problems with our telemetry system}

While the current 8/12-channel analog transmitters offer good-quality signals in virtually all conditions in our experimental setting, they provide more unstable/noisy signals than the digital transmitters because they are more sensitive to the RF physical environment changes in their close vicinity. Closer supervision is thus necessary, which is unpractical or even impossible at night during round-the-clock recording. We eventually anticipate replacing our analog transmitters by digital transmitters as soon as possible. In the meantime, we are currently optimizing the analog transmitters to be less sensitive to these physical environment changes.

Finally, the total weight of our 3 transmitters + batteries $(340 \mathrm{~g})$ represents a significant load for the youngest, tiniest preterm lambs, thus generating some discomfort in the standing position. Further improvements will be needed to decrease the size and weight of the overall system, now that we intend to study preterm lambs on a more frequent basis. In its current format, our radiotelemetry could be used in large animals such as cats, dogs, rabbits, swine, monkeys, sheep and goats, but is not adapted to the study of small rodents. 


\section{CONCLUSION}

Continuous telemetered recording of respiration has been made available commercially within the last 2 years, especially in response to FDA requirements for new drug applications. In our laboratory, we have rather progressively developed our own radio telemetry system over the last 15 years to fulfill our needs for polysomnographic recordings of neonatal breathing in ovine models. To our knowledge, the system presented herein allows the simultaneous recording of the highest number of physiological signals via telemetry, and has allowed us to gain a unique expertise in radio telemetry recording of breathing. Obviously, telemetered recording of breathing offers great promise for biomedical animal research and a worldwide spread of this technology is predictable in the forthcoming years.

\section{ACKNOWLEDGEMENTS}

The authors acknowledge the technical assistance of Jean-Philippe Gagné. This work is funded by the Canadian Institutes of Health (MOP 15558). Jean-Paul Praud is a member of the FRSQ-funded Clinical Research Center Étienne-Le Bel, Sherbrooke University Hospital, and the holder of the Canada Research Chair in Neonatal Respiratory Physiology. Marie-Laure Specq holds a doctoral scholarship from the Lung Association, Canadian Thoracic Society and a Merit scholarship for foreign students in University from the Ministère de l'Education, du Loisir et du Sport du Québec. 


\section{REFERENCES}

Al-Khalidi, F.Q., Saatchi, R., Burke, D., Elphick, H., Tan, S., 2011. Respiration rate monitoring methods: a review. Pediatr. Pulmonol. 46, 523-529.

Al-Qarawi, A.A., 2005. Immobilization (restraint) stress in desert sheep and goats, and the influence of pretreatment with xylazine or sodium betaine thereon. Pol. J. Vet. Sci. 8, 73-78.

Antier, D., Zhang, B.L., Mailliet, F., Akoka, S., Pourcelot, L., Sannajust, F., 1998. Effects of neonatal focal cerebral hypoxia-ischemia on sleep-waking pattern, ECoG power spectra and locomotor activity in the adult rat. Brain Res. 807, 29-37.

Authier, S., Haefner, P., Fournier, S., Troncy, E., Moon, L.B., 2010. Combined cardiopulmonary assessments with implantable telemetry device in conscious freely moving cynomolgus monkeys. J. Pharmacol. Toxicol. Methods 62, 6-11.

Baekey, D.M., Feng, P., Decker, M.J., Strohl, K.P., 2009. Breathing and sleep: measurement methods, genetic influences, and developmental impacts. ILAR J. 50, 248-261.

Brooks, D., Horner, R.L., Kozar, L.F., Waddell, T.K., Render, C.L., Phillipson, E.A., 1996. Validation of a telemetry system for long-term measurement of blood pressure. J. Appl. Physiol. 81, 1012-1018.

Butz, G.M., Davisson, R.L., 2001. Long-term telemetric measurement of cardiovascular parameters in awake mice: a physiological genomics tool. Physiol. Genomics 5, 89-97. 
Carreau, A-M., Patural, H., Samson, N., Doueik, A.A., Hamon, J., Fortier, P-H., Praud, J-P., 2011. Effects of Simulated Reflux Laryngitis on Laryngeal Chemoreflexes in Newborn Lambs. J. Appl. Physiol. In press.

Carrier, C.A., Elliott, T.B., Ledney, G.D., 2010. Real-time telemetric monitoring in wholebody 60Co gamma-photon irradiated rhesus macaques (Macaca mulatta). J. Med. Primatol. 39, 399-407.

Champeroux, P., Lala, P., Richard, S., 1994. Use of telemetry for electroencephalogram monitoring in conscious rabbits. Edinburgh: Br. Toxicol. Soc.

DeLorme, M.P., Moss, O.R., 2002. Pulmonary function assessment by whole-body plethysmography in restrained versus unrestrained mice. J. Pharmacol. Toxicol. Methods 47, 1-10.

Dilsaver, S.C., Overstreet, D.H., Peck, J.A., 1992. Measurement of temperature in the rat by rectal probe and telemetry yields compatible results. Pharmacol. Biochem. Behav. 42, 549-552.

Ednick, M.D., Pagala, M., Barakat, J.P., Nino, G., Shah, P., Cunningham, J.N.,Jr, Vaynblat, M., Kazachkov, M., 2007. Telemetric recording of intrapleural pressure. J. Surg. Res. 138, 10-14.

Eigenberg, R.A., Brown-Brandl, T.M., Nienaber, J.A., 2008. Sensors for dynamic physiological measurements. Comput. Electron. Agric. 62: 41-47. 
Gordon, C.J., Ali, J.S., 1984. Measurement of ventilatory frequency in unrestrained rodents using microwave radiation. Respir. Physiol. 56, 73-79.

Hegde, P., Singh, K., Chaplot, S., Shankaranarayana Rao, B.S., Chattarji, S., Kutty, B.M., Laxmi, T.R., 2008. Stress-induced changes in sleep and associated neuronal activity in rat hippocampus and amygdala. Neuroscience 153, 20-30.

Herzog, W., Stano, A., Leonard, T.R., 1993. Telemetry system to record force and EMG from cat ankle extensor and tibialis anterior muscles. J. Biomech. 26, 1463-1471.

Insalaco, G., Kuna, S.T., Costanza, B.M., Catania, G., Cibella, F., Bellia, V., 1991. Thyroarytenoid muscle activity during loaded and nonloaded breathing in adult humans. J. Appl. Physiol. 70, 2410-2416.

Kant, G.J., Pastel, R.H., Bauman, R.A., Meininger, G.R., Maughan, K.R., Robinson, T.N.,3rd, Wright, W.L., Covington, P.S., 1995. Effects of chronic stress on sleep in rats. Physiol. Behav. 57, 359-365.

Kawai, N., Tanaka, E., Langenbach, G.E., van Wessel, T., Sano, R., van Eijden, T.M., Tanne, K., 2008. Jaw-muscle activity changes after the induction of osteoarthrosis in the temporomandibular joint by mechanical loading. J. Orofac. Pain 22, 153-162.

Kearney, K., Metea, M., Gleason, T., Edwards, T., Atterson, P., 2010. Evaluation of respiratory function in freely moving Beagle dogs using implanted impedance technology. J. Pharmacol. Toxicol. Methods 62, 119-126. 
Kramer, K., van Acker, S.A., Voss, H.P., Grimbergen, J.A., van der Vijgh, W.J., Bast, A., 1993. Use of telemetry to record electrocardiogram and heart rate in freely moving mice. J. Pharmacol. Toxicol. Methods 30, 209-215.

Kramer, K., Kinter, L., Brockway, B.P., Voss, H.P., Remie, R., Van Zutphen, B.L., 2001. The use of radio telemetry in small laboratory animals: recent advances. Contemp. Top. Lab. Anim. Sci. 40, 8-16.

Kramer, K., Kinter, L.B., 2003. Evaluation and applications of radio telemetry in small laboratory animals. Physiol. Genomics 13, 197-205.

Layton, R.C., Brasel, T., Gigliotti, A., Barr, E., Storch, S., Myers, L., Hobbs, C., Koster, F., 2011. Primary pneumonic plague in the African Green monkey as a model for treatment efficacy evaluation. J. Med. Primatol. 40, 6-17.

Letourneau, P., Dumont, S., Kianicka, I., Diaz, V., Dorion, D., Drolet, R., Praud, J-P., 1999. Radio telemetry system for apnea study in lambs. Respir. Physiol. 116, 85-93.

Letourneau, P., Praud, J-P., 2003. A radio telemetry system for polysomnographic recordings in lambs. Methods 30, 115-121.

Livezey, G.T., Sparber, S.B., 1990. Hyperthermia sensitizes rats to cocaine's proconvulsive effects and unmasks EEG evidence of kindling after chronic cocaine. Pharmacol. Biochem. Behav. 37, 761-767. 
McLeod, C.N., Harding, R., Johnson, P., McClelland, M.E., Whyte, P.L., 1979. Studies on the control of respiration and behaviour during development in ewe-reared lambs. Biotelem. Patient Monit. 6, 171-175.

Meile, T., Zittel, T.T., 2002. Telemetric small intestinal motility recording in awake rats: a novel approach. Eur. Surg. Res. 34, 271-274.

Mills, P.A., Huetteman, D.A., Brockway, B.P., Zwiers, L.M., Gelsema, A.J., Schwartz, R.S., Kramer, K., 2000. A new method for measurement of blood pressure, heart rate, and activity in the mouse by radio telemetry. J. Appl. Physiol. 88, 1537-1544.

Mumford, H., Wetherell, J.R., 2001. A simple method for measuring EEG in freely moving guinea pigs. J. Neurosci. Methods 107, 125-130.

Murphy, D.J., Renninger, J.P., Gossett, K.A., 1998. A novel method for chronic measurement of pleural pressure in conscious rats. J. Pharmacol. Toxicol. Methods 39, $137-141$

Murphy, D.J., Renninger, J.P., Schramek, D., 2010. Respiratory inductive plethysmography as a method for measuring ventilatory parameters in conscious, nonrestrained dogs. J. Pharmacol. Toxicol. Methods 62, 47-53.

Nakajima, M., Sakai, T., Mizumoto, A., Itoh, Z., 1996. Development of a new telemetry recording system for measuring of gastrointestinal contractile activity in unrestrained and conscious small animals. J. Smooth Muscle Res. 32, 1-7. 
Praud, J-P., Samson, N., Moreau-Bussiere, F., 2006. Laryngeal function and nasal ventilatory support in the neonatal period. Paediatr. Respir. Rev. 7 Suppl 1, S180-2.

Reix, P., Dumont, S., Duvareille, C., Cyr, J., Moreau-Bussiere, F., Arsenault, J., Praud, J-P., 2005. Monitoring pulse oximetry via radio telemetry in freely-moving lambs. Respir. Physiol. Neurobiol. 147, 65-72.

Renolleau, S., Letourneau, P., Niyonsenga, T., Praud, J-P., 1999. Thyroarytenoid muscle electrical activity during spontaneous apneas in preterm lambs. Am. J. Respir. Crit. Care Med. 159, 1396-1404.

Roy, B., Samson, N., Moreau-Bussiere, F., Ouimet, A., Dorion, D., Mayer, S., Praud, JP., 2008. Mechanisms of active laryngeal closure during noninvasive intermittent positive pressure ventilation in nonsedated lambs. J. Appl. Physiol. 105, 1406-1412.

Russell, W.M.S., Burch, R.L., 1959. The Principles of Humane Experimental Technique. London: Methuen.

Samson, N., St-Hilaire, M., Nsegbe, E., Reix, P., Moreau-Bussiere, F., Praud, J-P., 2005. Effect of nasal continuous or intermittent positive airway pressure on nonnutritive swallowing in the newborn lamb. J. Appl. Physiol. 99, 1636-1642.

Samson, N., Lafond, J.R., Moreau-Bussiere, F., Reix, P., Praud, J-P., 2007.

Cricothyroid muscle electrical activity during respiration and apneas in lambs. Respir. Physiol. Neurobiol. 155, 147-155. 
Samson, N., Roy, B., Ouimet, A., Moreau-Bussiere, F., Dorion, D., Mayer, S., Praud, JP., 2008. Origins of the inhibiting effects of nasal CPAP on nonnutritive swallowing in newborn lambs. J. Appl. Physiol. 105, 1083-1090.

Sei, H., Morita, Y., 1996. Acceleration of EEG theta wave precedes the phasic surge of arterial pressure during REM sleep in the rat. Neuroreport 7, 3059-3062.

St-Hilaire, M., Samson, N., Nsegbe, E., Duvareille, C., Moreau-Bussiere, F., Micheau, P., Lebon, J., Praud, J-P., 2007. Postnatal maturation of laryngeal chemoreflexes in the preterm lamb. J. Appl. Physiol. 102, 1429-1438.

St-Hilaire, M., Duvareille, C., Avoine, O., Carreau, A-M., Samson, N., Micheau, P., Doueik, A., Praud, J-P., 2010. Effects of postnatal smoke exposure on laryngeal chemoreflexes in newborn lambs. J. Appl. Physiol. 109, 1820-1826.

Suzuki, S., Matsui, T., Kawahara, H., Gotoh, S., 2009. Development of a noncontact and long-term respiration monitoring system using microwave radar for hibernating black bear. Zoo Biol. 28, 259-270.

Tang, X., Xiao, J., Parris, B.S., Fang, J., Sanford, L.D., 2005. Differential effects of two types of environmental novelty on activity and sleep in BALB/CJ and C57BL/6J mice. Physiol. Behav. 85, 419-429.

Tang, X., Yang, L., Sanford, L.D., 2007. Interactions between brief restraint, novelty and footshock stress on subsequent sleep and EEG power in rats. Brain Res. 1142, 110118. 
Trelease, R.B., Sieck, G.C., Harper, R.M., 1982. A new technique for acute and chronic recording of crural diaphragm EMG in cats. Electroencephalogr. Clin. Neurophysiol. 53, 459-462.

Warren, R., Lockman, H., Barnewall, R., Krile, R., Blanco, O.B., Vasconcelos, D., Price, J., House, R.V., Bolanowksi, M.A., Fellows, P., 2011. Cynomolgus macaque model for pneumonic plague. Microb. Pathog. 50, 12-22. 
Table 1: Comparison of physiological signals recorded by our telemetry system with commercially available systems offering telemetered recording of respiration for biomedical research in animals

\begin{tabular}{|c|c|c|c|c|c|c|c|c|}
\hline & RIP & ECG & $\begin{array}{l}\text { Blood } \\
\text { pressure }\end{array}$ & Activity & Temp & EEG, EMGs & $\begin{array}{c}\text { EOG, nasal flow, } \mathrm{SatO}_{2}, \\
\text { respiratory pressures }\end{array}$ & Transmitters \\
\hline DSI & $\begin{array}{c}\text { Yes } \\
\text { (Jet system) }\end{array}$ & Yes & $\begin{array}{c}\text { Yes } \\
\text { invasive }\end{array}$ & $\begin{array}{c}\text { Yes } \\
\text { (Actigraphy) }\end{array}$ & Yes & Yes & No & $\begin{array}{l}\text { Implanted } \\
\text { (except Jet) }\end{array}$ \\
\hline MK & $\begin{array}{c}\text { Yes } \\
\text { (Emkapack) }\end{array}$ & Yes & $\begin{array}{l}\text { Yes non- } \\
\text { invasive }\end{array}$ & $\begin{array}{c}\text { Yes } \\
\text { (Actigraphy) }\end{array}$ & Yes & No & No & Not implanted \\
\hline $\begin{array}{l}\text { Our } \\
\text { system }\end{array}$ & Yes & Yes & $\begin{array}{c}\text { Yes } \\
\text { invasive }\end{array}$ & Yes (video) & No & Yes & Yes & Not implanted \\
\hline
\end{tabular}

RIP: respiratory inductance plethysmograph; ECG: electrocardiogram; Temp: temperature; EEG: electroencephalogram; EOG: electrooculogram; EMG: electromyographic activity of respiratory and upper airway muscles; $\mathbf{S a t O}_{2}$ : hemoglobin $\mathrm{O}_{2}$ saturation (pulse oximetry); DSI: Data Sciences International; MK: Emka Technologies 


\section{FIGURE LEGENDS}

\section{Figure 1: Block diagram of the transmitters}
(A) 12-channel analog, (B) 8-channel digital and (C) Masimo pulse oximetry transmitters.

\section{Figure 2: Block diagram of the receivers}

(A) 12-channel analog, (B) 8-channel digital and (C) Masimo pulse oximetry receivers.

Figure 3: Respiration during quiet sleep in a newborn lamb

ECoG (electrocorticogram), EOG (electrooculogram), TA [electrical activity (EMG) of the thyroarytenoid muscle, a glottal constrictor muscle], fTA (integrated TA EMG) and ECG (electrocardiogram) were transmitted via our 12-channel analog system. Thorax and Abdomen (thoracic and abdominal respiratory movements recorded via respiratory inductance plethysmography, with inspiration upward), AP (arterial pressure, $\mathrm{mmHg}$, recorded via a liquid-filled carotid catheter-transducer system) were transmitted via our 8-channel digital system; $\mathbf{S p O}_{2}$ (oxygen hemoglobin saturation, \%) and Pulse wave (pulse wave recorded by a Masimo pulse oximetry probe) were transmitted via our 4channel Masimo digital system. 
Figure 4: Respiratory activity of several upper airway muscles in a newborn lamb during wakefulness

See figure 3 for abbreviations. Genio (genioglossus muscle, an upper airway dilator) and JGenio (integrated Genio EMG), LP (EMG of the levator palatini muscle, a pharyngeal muscle that elevates the soft palate) and JLP (integrated LP EMG), TA and $\int$ TA, CT and $\int \mathbf{C T}$ were transmitted via our 12-channel analog system. Thorax and Abdomen were transmitted via our 8-channel digital system. The regular inspiratory EMG activity of Genio and LP muscles observed during wakefulness in this recording is unusual in the newborn lamb (usually absent).

Figure 5: Respiratory activity of glottal muscles and diaphragm during quiet wakefulness in a newborn lamb

See figure 3 for abbreviations. TA and $\int \mathbf{T A}, \mathbf{C T}$ and $\int \mathbf{C T}$, Di (diaphragm) and $\int \mathbf{D i}$, ECG, ECoG and EOG were transmitted via our 12-channel analog system. F (nasal flow recorded via a thermocouple, with inspiration upward) was transmitted via our 8-channel digital system. A typical respiratory activity of glottal muscles is illustrated, with glottal dilator EMG (CT) in inspiration ( $†$ ) and late expiration $(\ddagger)$, and glottal constrictor EMG $(\mathrm{TA})$ in early expiration $\left({ }^{*}\right)$. 
Figure 1
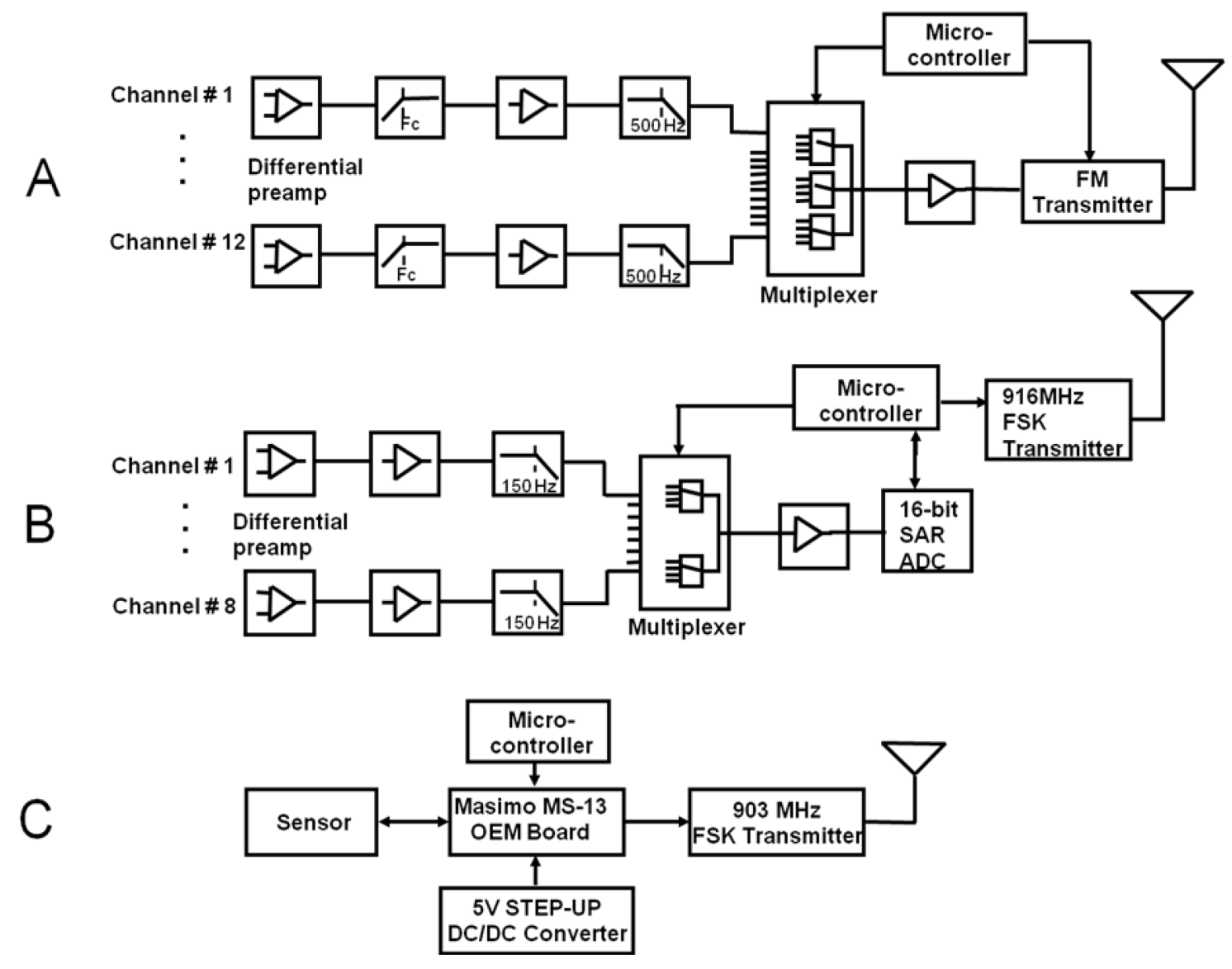
Figure 2

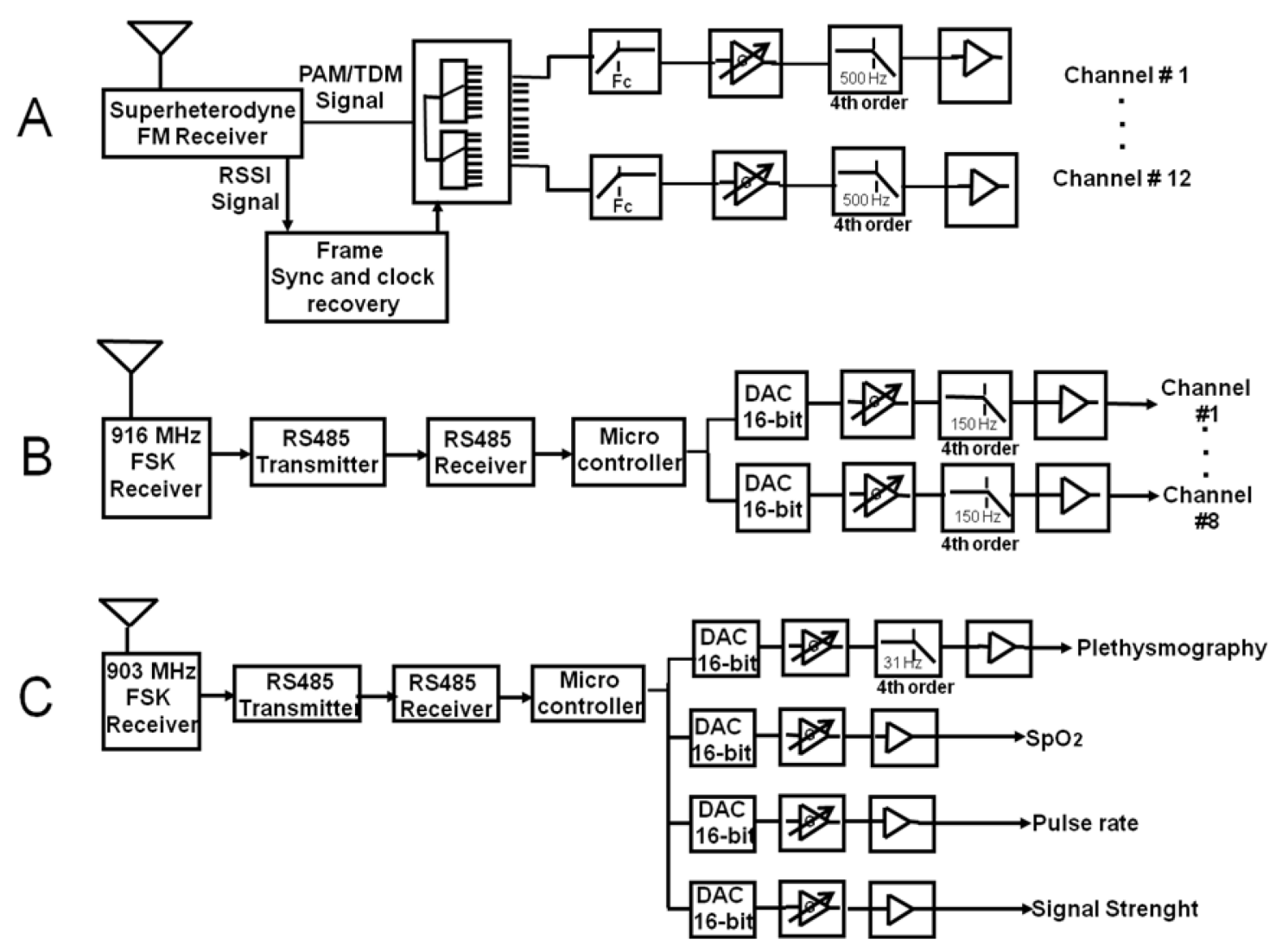


Figure 3

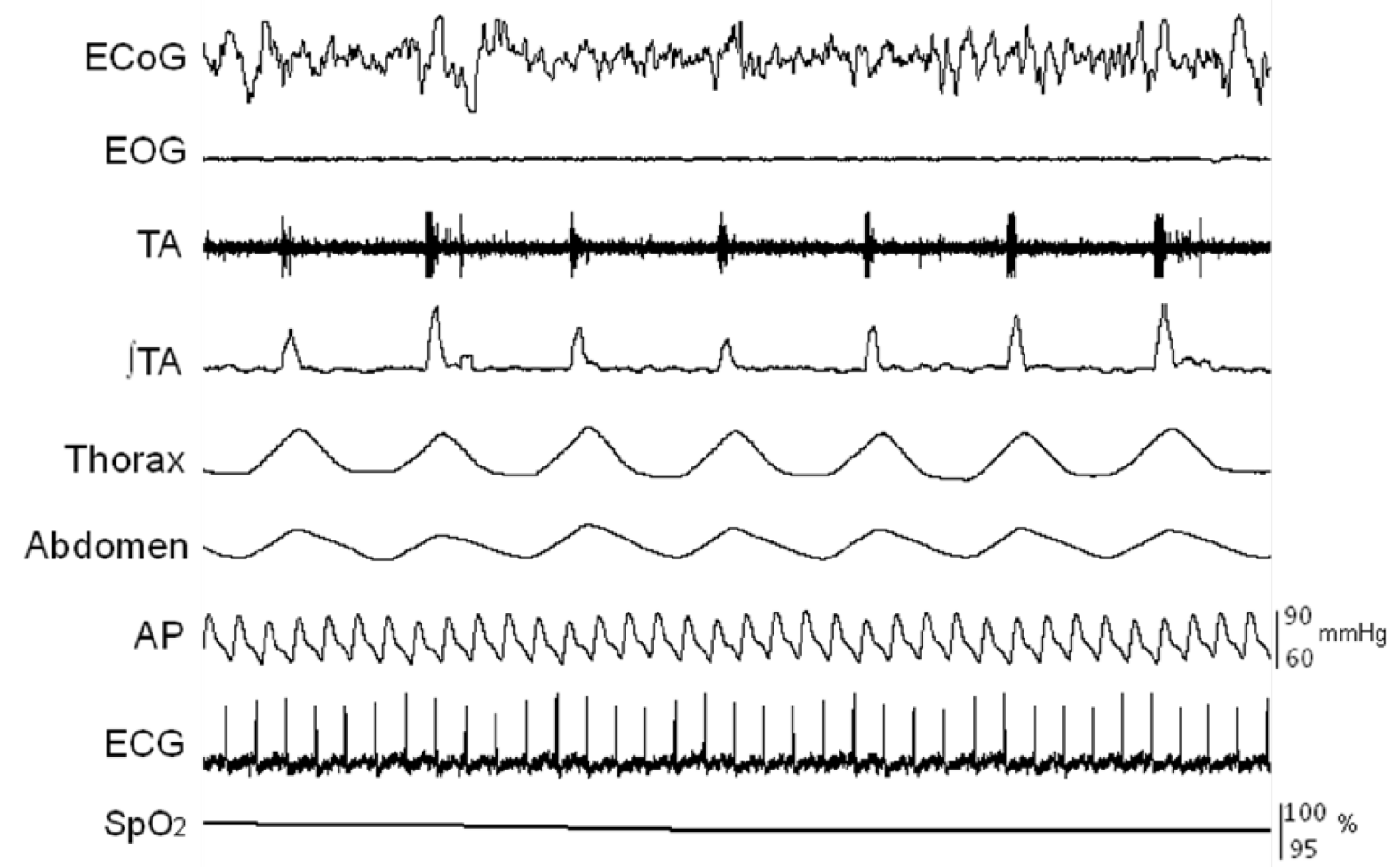

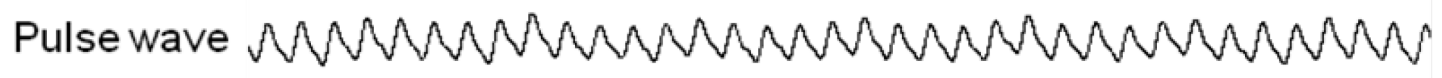


Figure 4

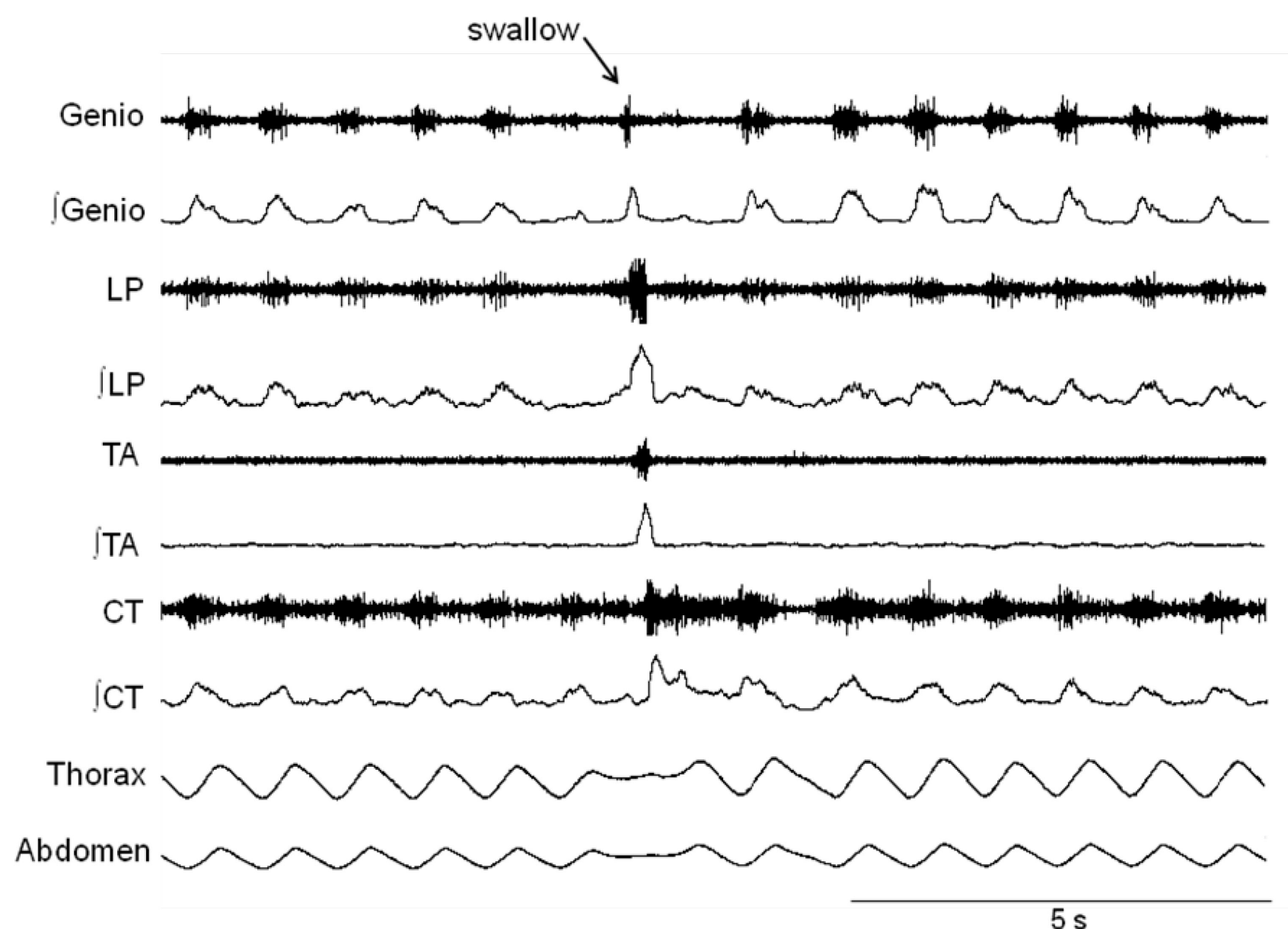


Figure 5

TA TTA bun

CT
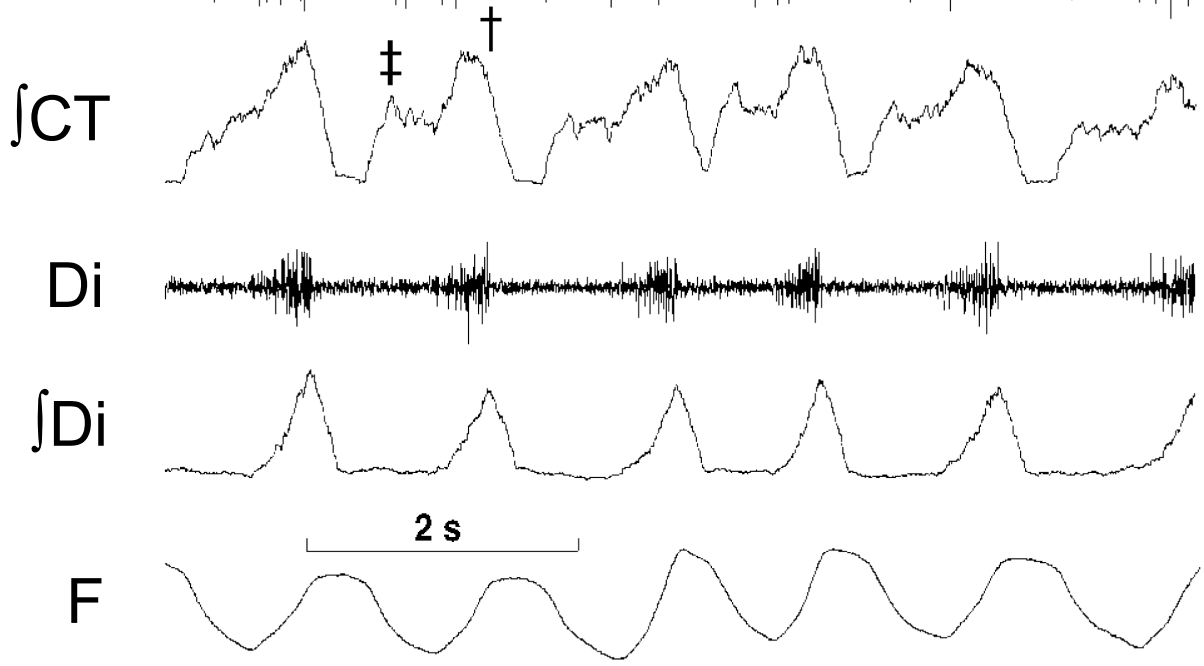

ECG

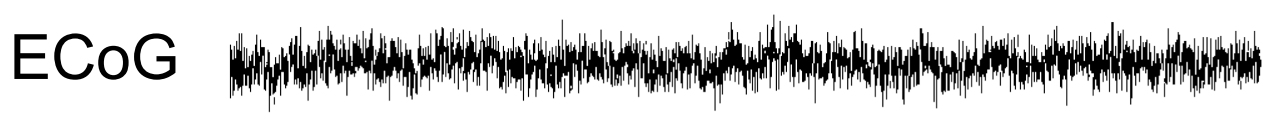

EOG 